\title{
ANÁLISIS TECNO-TIPOLÓGICO DE INSTRUMENTOS LÍTICOS DEL SITIO ARCAICO TEMPRANO IPILLA 2 EN LOS ANDES DE ARICA, CHILE*
}

\author{
TECHNO-TYPOLOGICAL ANALYSIS OF LITHIC TOOLS FROM IPILLA 2 \\ AN EARLY ARCHAIC SITE IN THE ANDES OF ARICA, CHILE
}

\author{
Katherine A. Herrera1, Paula C. Ugalde ${ }^{2}$, Daniela Osorio², José M. Capriles ${ }^{1,3}$, \\ Salomón Hocsman y Calogero M. Santoro ${ }^{1,2,+}$
}

\begin{abstract}
El estudio de la tecnología lítica en los Andes se inició con la identificación tipológica de puntas de proyectil para definir secuencias cronológico-culturales de sociedades de cazadores recolectores. Análisis tecno-tipológicos como el que se presenta en este trabajo, tratan de mostrar que las morfologías de instrumentos líticos no son estáticas pues varían de acuerdo a su uso, mantenimiento y reciclaje. En este estudio, exploramos y caracterizamos la variabilidad morfológica del instrumental lítico del sitio Ipilla 2, un campamento abierto del Arcaico Temprano (9.670-9.541 cal. a.p.), ubicado en los Andes de Arica (3.400 msm), norte de Chile. Los resultados sugieren que los instrumentos fueron intensamente mantenidos para extender su vida útil, lo que alteró los diseños originales. Otro proceso tecnológico incluyó la manufactura secuencial de distintos filos en un mismo instrumento. Estos resultados aportan a la comprensión de los modos de vida de las sociedades de cazadores recolectores andinos y muestran que, metodológicamente, las formas tipológicas deben considerarse desde una perspectiva dinámica para convertirse en una herramienta analítica más eficaz.
\end{abstract}

Palabras claves: Análisis líticos tecno-tipológicos, cazadores recolectores andinos, Arcaico Temprano.

The study of lithic technology in the Andes began with the classification of projectile point typologies to define chronological and cultural sequences of hunter-gatherer societies. Techno-typological analysis such as the one presented here, try to show that lithic morphologies are not static as they vary in relation to their use, maintenance and recycling. In this study we explore and characterize the technological and typological variability of stone tools from Ipilla 2, an Early Archaic open-air camp (9,670-9,541 cal. BP) located in the Andes of Arica (3,400 $\mathrm{m}$ asl), northern Chile. The results show that tools were intensely maintained to extend their use-life, which transformed their original designs. Another technological process included the sequential manufacture of different edges in the same instrument. These results contribute to understanding the lifestyles of Andean hunter-gatherer societies and show that methodologically, lithic typologies must be considered from a dynamic perspective to become a more effective analytic tool.

Key words: Techno-typological lithic analysis, Andean hunter-gatherers, Early Archaic.

Los análisis tipológicos de artefactos líticos en los Andes son una herencia del desarrollo de la arqueología del Viejo Mundo (Bate 1974; Montane 1972; Núñez 1983; Odell 1996; Swanson 1975). Estos estudios adquirieron gran importancia para definir la cronología y distribución espacial de diversos grupos de cazadores recolectores identificados a través de formas diagnósticas de puntas de proyectil (i.e. El Jobo, Ayampitín, Paiján, Cola de Pescado) y, ocasionalmente, otros instrumentos

* Although this article was not presented at the "Early Lithic Technologies: Beyond Regional Projectile Point Typologies" Symposium at the 77th SAA Meeting Memphis, Tennessee, April 2012, the guest editors Kurt Rademaker and César Méndez invited us to be part of this issue of Chungara Revista de Antropología Chilena.

1 Instituto de Alta Investigación, Universidad de Tarapacá, Antofagasta 1520, Arica, Chile. herreragodoy@ hotmail.com; calogero_santoro@yahoo.com

2 Centro de Investigaciones del Hombre en el Desierto (CIHDE), Avenida General Velásquez 1775, Arica, Chile. pugalde@cihde.cl; dosorio@cihde.cl

3 Departamento de Antropología, Universidad de Tarapacá, 18 de Septiembre 2222, Casilla 6-D, Arica, Chile. jmcapriles@gmail.com

4 CONICET - Instituto Superior de Estudios Sociales, CONICET-UNT - Instituto de Arqueología y Museo, Facultad de Ciencias Naturales e Instituto Miguel Lillo, Universidad Nacional de Tucumán, San Martín 1545 (4000), San Miguel de Tucumán, Argentina. shocsman@hotmail.com

+ Autor correspondiente. 
como raspadores, raederas y cuchillos (Bird 1965; Cigliano 1962; Cruxent 1962; Cruxent y Rouse 1956; Dillehay 1999; Dillehay et al. 1992; González 1960; Lanning 1973; Lanning y Hammel 1961; Le Paige 1970; Ravines 1972; Santoro y Núñez 1987; Taddei 1981, 1987; Willey 1966; Willey y Phillips 1958). A pesar de evaluaciones críticas respecto de su validez cronológica y cultural (Bate 1974; Bird 1965; Montane 1972), ciertas tipologías de puntas de proyectil se siguen utilizando como indicadores espacio-temporales en la prehistoria andina (Capriles et al. 2011; Dillehay 2000; Klink y Aldenderfer 2005).

Sin restar importancia al aporte de esta corriente teórico-metodológica, los estudios centrados exclusivamente en aspectos meramente descriptivos de las formas de los instrumentos limitan la posibilidad de conocer los procesos técnicos de elaboración, el saber hacer del tallador, la influencia de la calidad y disponibilidad de las materias primas, los efectos provocados por distintos procesos de talla en las morfologías de los artefactos y otras transformaciones aplicadas a los instrumentos a lo largo de su vida útil. Los análisis tecno-tipológicos, en cambio, ayudan a comprender los procesos de elaboración, uso, reutilización y descarte de los instrumentos líticos, y permiten abordar la variabilidad morfológica e historia de vida de los mismos (Hocsman 2006a, 2009, 2010; MalinskyBuller et al. 2011). Desde esta perspectiva las formas y funciones de los instrumentos no fueron estáticas, sino que por el contrario formaron parte de procesos tecnológicos dinámicos y variables (Andrefsky 2005, 2008; Aschero 1975, 1983; Aschero y Hocsman 2004; Bate 1971; Inizan et al. 1999; Nelson 1991; Schlanger 2005; Soressi y Geneste 2005). Los análisis tecno-tipológicos se han desarrollado especialmente en Argentina (Aschero et al. 2011; Borrazzo 2012; Franco 2004; Hocsman 2006b, 2010; Hoguin y Yacobaccio 2012), mientras que en el norte de Chile y regiones vecinas ha prevalecido la corriente tipológica "tradicional", con algunas excepciones (De Souza 2004; Osorio et al. 2011).

El objetivo de este trabajo es mostrar la factibilidad y utilidad metodológica del análisis tecno-tipológico de artefactos líticos en el contexto del sistema social del que formaron parte; en este caso los cazadores recolectores Arcaico Temprano de la zona andina de Arica. A modo de hipótesis, proponemos que las formas y funciones de los instrumentos de Ipilla 2 no se corresponden únicamente a morfologías estáticas. Por el contrario, sus formas finales descartadas son el resultado del dinámico proceso tecnológico que formó parte de la historia de los artefactos (Aschero 1975, 1983; Aschero y Hocsman 2004; Nelson 1991).

\section{Materiales y Métodos}

Ipilla se localiza en la quebrada La Higuera, a $3.400 \mathrm{msm}$ y a unos $11 \mathrm{~km}$ al suroeste del pueblo de Tignamar en el piso ecológico conocido como puna (Mujica et al. 2015), al sureste de Arica (Figura 1). Ipilla 2 es un sitio a cielo abierto que abarca una superficie aproximada de $800 \mathrm{~m}^{2}$, emplazado sobre una estrecha terraza fluvial adyacente a una paleovertiente (Rech 2001; Ugalde et al. 2012). El borde oeste del sitio está cortado por escorrentías que han expuesto la estratigrafía arqueológica, lo que se repite en el borde sureste fuertemente erosionado por el canal principal de la quebrada. Desde ese perfil expuesto se obtuvieron las primeras tres dataciones

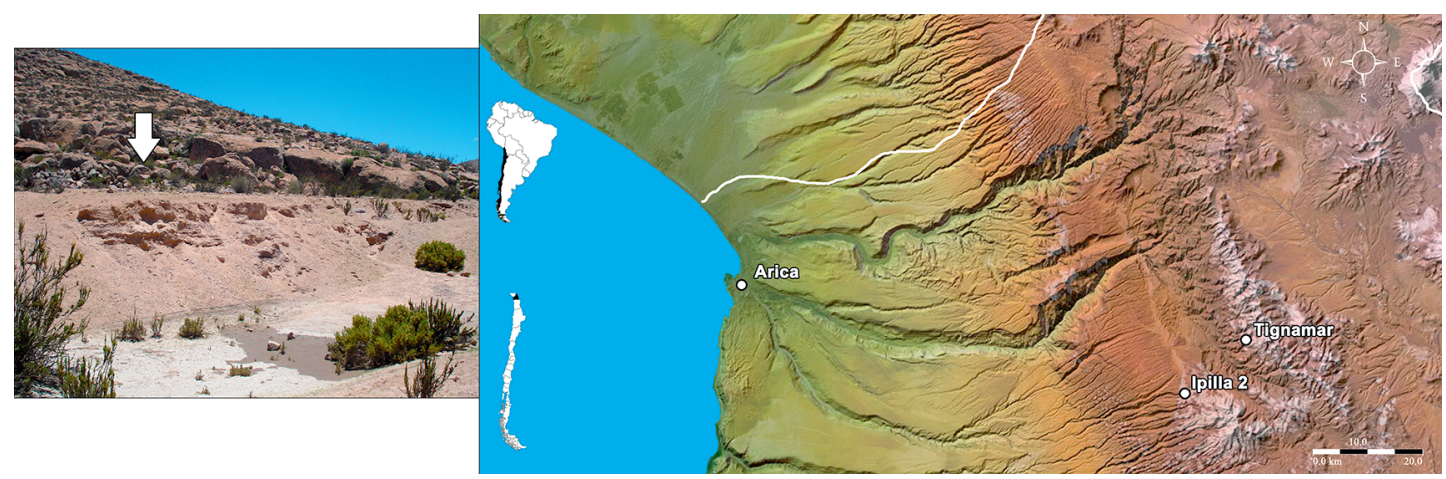

Figura 1. Ubicación del sitio Ipilla 2 con el pueblo de Tignamar y la ciudad de Arica como referencia. Location of the site Ipilla 2 with the town of Tignamar and the city of Arica shown for reference. 
radiocarbónicas en torno a los 8.800 años (Santoro et al. 2011; Ugalde et al. 2012). Por el noreste, el sitio está delimitado por un escarpado rocoso que pudo servir de refugio a cazadores recolectores. En la parte central de Ipilla 2 se ubica una estructura rectangular pircada de data desconocida, que podría estar relacionada a la enorme manga de captura de animales (chacu) ubicada en la ladera sur de Quebrada La Higuera, frente al sitio. Por esa misma banda se registró otro perfil expuesto que incluye un estrato con evidencias arqueológicas y que corresponde al sitio Ipilla 1, fechado en 10.191 123 años cal. a.p. (AA34477, 9090 \pm 75 a.p.) (Rech 2001).

La importancia del emplazamiento radica en la surgencia de una vertiente de agua dulce vigente durante el Holoceno Temprano (Rech 2001) y su proximidad a Pampa Oxa, una pradera hacia el norte que en aquella época debió tener mayor cantidad de recursos forrajeros para animales silvestres como guanacos y vicuñas (Mujica et al. 2015). Si bien Ipilla y sus inmediaciones no presenta en la actualidad condiciones para actividades agrícolas o pastoriles, los habitantes de Tignamar recordaban que el chacu, ubicado en la ladera sur frente a Ipilla, habría sido utilizado hasta comienzos del siglo veinte para acorralar burros salvajes, lo que coincide con la fase final de un período de mayor pluviosidad en la zona (Mujica et al. 2015).

El material arqueológico analizado, se obtuvo de la excavación de una cuadrícula de 2 x 1,30 m en el centro del sitio, cuya estratigrafía alcanzó $1,11 \mathrm{~m}$ de profundidad y fue separada en 15 estratos de los cuales, solamente los primeros 13 presentaban actividad humana. La ocupación más antigua corresponde al período Arcaico Temprano y comprende los estratos 6 al 13 (ubicados entre ca. 44 a $94 \mathrm{~cm}$ de profundidad), con ocho dataciones de ${ }^{14} \mathrm{C}$ AMS, que fluctúan entre 9.670 y 9.541 años cal. a.p., con algunas inversiones estratigráficas como muestra la Tabla 1. Para los estratos superiores se obtuvo un fechado en el estrato 5 que corresponde al período Formativo.

La estrecha dispersión temporal de los ocho fechados del Arcaico Temprano muestra que el sitio fue ocupado por un período de aproximadamente 129 años. Un modelo de combinación estadística descarta la posibilidad de que estas fechas correspondan a un solo evento depositacional $\left(\chi^{2}=20,146\right.$, d.f. $=$ $7, p<0,05$, Bronk Ramsey y Lee 2013, y curva de calibración SHCAL13, Hogg et al. 2013). Esta sección de la estratigrafía fue seguida por una discordancia erosiva caracterizada por una transición abrupta de sedimentos finos (estrato 6), a gravas fluviales (estrato 5) que pudo ser provocada por uno o más eventos fluviales que eliminaron una porción de la estratigrafía (Santoro et al. 2011; Ugalde et al. 2012). Esto explicaría la superposición de las ocupaciones de época Formativa sobre los niveles Arcaico Temprano y la ausencia de ocupaciones Arcaico Medio y Tardío.

Se realizó un análisis tecno-tipológico de todo el material lítico recuperado de los estratos 6 a 13 con excepción de los microdesechos $(<7 \mathrm{~mm})$ que sólo fueron contabilizados y clasificados de acuerdo a su conservación (completos/fracturados). El análisis de los derivados de talla se centró en establecer secuencias de reducción (Andrefsky 2005; Bate 1971). El análisis de instrumentos siguió los

Tabla 1. Fechados radiocarbónicos AMS del sitio Ipilla 2. La calibración y el modelo de combinación se realizó en Oxcal 4.2.3 (Bronk Ramsey y Lee 2013) empleando la curva de calibración SHCAL13 (Hogg et al. 2013).

Radiocarbon AMS dating from Ipilla 2 site. Calibration and combination model was performed with Oxcal 4.2.3 (Bronk Ramsey \& Lee 2013), using the SHCAL13 calibration curve (Hogg et al. 2013).

\begin{tabular}{|c|c|c|c|c|}
\hline Código de muestra & Años ${ }^{14} \mathrm{C}$ & Años cal. a.p. $(2 \sigma)$ & Material & Estrato \\
\hline UCIAMS-77763 & $2075 \pm 35$ & 2090-1904 & Carbón & 5 \\
\hline UCIAMS-84348 & $8675 \pm 25$ & $9670-9535$ & Carbón & 6 \\
\hline UCIAMS-77764 & $8635 \pm 40$ & $9665-9492$ & Carbón & 7 \\
\hline UCIAMS-77765 & $8600 \pm 40$ & $9599-9472$ & Carbón & 8 \\
\hline UCIAMS-77766 & $8695 \pm 40$ & $9730-9532$ & Carbón & 9 \\
\hline UCIAMS-77767 & $8690 \pm 40$ & $9703-9531$ & Carbón & 10 \\
\hline UCIAMS-77768 & $8730 \pm 35$ & $9762-9542$ & Carbón & 11 \\
\hline UCIAMS-77769 & $8825 \pm 40$ & $10125-9565$ & Carbón & 12 \\
\hline UCIAMS-77770 & $8695 \pm 40$ & $9730-9532$ & Carbón & 13 \\
\hline \multicolumn{2}{|c|}{ Modelo combinado (estratos 6-13) } & $9670-9541$ & & \\
\hline
\end{tabular}


preceptos de la morfología descriptiva que disocia los componentes de la morfología de un objeto a fin de establecer sus particularidades (Aschero 1975, 1983; Aschero y Hocsman 2004; Brézillon 1983; Hocsman 2006b). Además, se realizó una definición general de funciones de los instrumentos a partir de la identificación de huellas de uso con microscopio estereoscópico (45x) (Lewenstein 1990; Mirambell 1994; Semenov 1981).

\section{Resultados}

Entre los estratos 6 al 13 se obtuvo un total de 9.210 piezas que se analizaron como un solo conjunto dado el corto período de tiempo al que están asociados. De ese total, solamente 18 corresponden a instrumentos, 1.870 a derivados de talla y 7.322 a microdesechos (Tabla 2).

Las materias primas fueron identificadas macroscópicamente por el geólogo Esteban Briones y se comportan de la siguiente manera. Destaca el uso de sílice $(79,4 \%)$, de textura homogénea, colores claros y de buena calidad para tallar, seguido de otras seis materias primas, entre las que predomina un jaspe de muy buena calidad (7,1\%) (Tabla 3). Existe clara correspondencia entre las materias primas representadas en los desechos de talla y aquellas con las que se manufacturaron los instrumentos, lo que significa que éstos fueron retocados in situ. Respecto de la procedencia de estas materias primas, tanto el sílice como el jaspe podrían provenir de afloramientos disponibles localmente. De las otras no tenemos conocimiento.

\section{Derivados de talla}

Los derivados de talla corresponden mayormente a desechos de retoque $(41,6 \%)$ por sobre las lascas $(21,9 \%)$, los desechos bifaciales $(10,5 \%)$ y las láminas $(1,8 \%)$. Los atributos de los derivados de talla incluyen: (a) ausencia de corteza en un 98,5\% de los casos; (b) tamaño pequeño en un 80,3\% de los derivados completos (longitud promedio $=14,3 \pm 7$ $\mathrm{mm})$; (c) presencia de aristas mixtas $(24,3 \%)$; (d) talones planos y pequeños (36\%), talones facetados $(0,8 \%)$ y diedros $(0,2 \%)$. Todos estos atributos, considerados en su conjunto, sugieren que los desechos fueron producidos durante la formatización

Tabla 2. Frecuencias y porcentajes de artefactos líticos recuperados de los niveles 6 a 13, sitio Ipilla 2. Frequencies and proportions of lithic artifacts recovered from levels 6 to 13, Ipilla 2 site.

\begin{tabular}{|c|c|c|}
\hline Categorías & $\mathrm{N}$ & $\%$ \\
\hline \multicolumn{3}{|l|}{ Derivados de talla } \\
\hline Lasca & 409 & 21,9 \\
\hline Lámina & 34 & 1,8 \\
\hline Fragmento & 371 & 19,8 \\
\hline Desecho de retoque & 778 & 41,6 \\
\hline Desecho bifacial & 197 & 10,5 \\
\hline Indeterminado & 81 & 4,3 \\
\hline Subtotal & 1.870 & 100 \\
\hline \multicolumn{3}{|l|}{ Instrumentos } \\
\hline \multicolumn{3}{|l|}{ No formatizados } \\
\hline Cuchillo de filo natural con rastros complementarios & 3 & 16,7 \\
\hline \multicolumn{3}{|l|}{ Formatizados } \\
\hline Cuchillo de filo retocado & 1 & 5,6 \\
\hline Percutor de arista formatizada & 1 & 5,6 \\
\hline Denticulado en bisel oblicuo/abrupto & 1 & 5,6 \\
\hline Bifaz & 1 & 5,6 \\
\hline Preforma de punta de proyectil & 1 & 5,6 \\
\hline Puntas de proyectil & 3 & 16,5 \\
\hline Instrumento compuesto & 1 & 5,6 \\
\hline Cortante de filo retocado + filo formatizado pasivo sobre punta de proyectil reciclada & 1 & 5,6 \\
\hline Fragmentos no diferenciados de artefactos formatizados & 5 & 27,6 \\
\hline Subtotal & 18 & 100 \\
\hline Micro-desechos $(<7 \mathrm{~mm})$ & 7.322 & 100 \\
\hline Total & 9.210 & 100 \\
\hline
\end{tabular}


Tabla 3. Frecuencias y porcentajes de materias primas de los artefactos líticos analizados de Ipilla 2.

Frequencies and proportions of raw materials from analyzed lithic artifacts of Ipilla 2.

\begin{tabular}{lrrrr}
\hline \multirow{2}{*}{ Materia prima } & \multicolumn{2}{c}{ Derivados de talla } & \multicolumn{2}{c}{ Instrumentos } \\
\cline { 2 - 5 } & \multicolumn{1}{c}{$\mathrm{N}$} & \multicolumn{1}{c}{$\%$} & $\mathrm{~N}$ & $\%$ \\
\hline Sílice & 1.485 & 79,4 & 13 & 72,2 \\
Arenisca & 29 & 1,6 & 1 & 5,6 \\
Sílice-jaspe & 43 & 2,3 & & \\
Arenisca-jaspe & 38 & 2 & & \\
Andesita & 22 & 1,2 & & \\
Jaspe & 132 & 7,1 & 4 & 22,2 \\
Obsidiana & 7 & 0,4 & & \\
Indeterminado & 114 & 6,1 & & \\
Total & 1.870 & 100 & 18 & 100 \\
\hline
\end{tabular}

final y/o la reactivación de instrumentos. Lo que sugiere que en el sitio están presentes principalmente etapas finales de la secuencia de reducción y, en menor medida, etapas medias. Además, se observan algunas técnicas específicas de extracción que incluyen preparación de borde adyacente $(4,1 \%)$ y preparación de talones (rebajados y machacados). Finalmente la muestra contiene baja frecuencia de bisagra (o charnela) en anverso $(0,5 \%)$ y reverso $(1,3 \%)$. Esto puede deberse a dos factores no excluyentes entre sí. Por un lado, la buena calidad de la materia prima y por otro, la habilidad de los talladores quienes cometieron pocos errores a pesar de la complejidad de los instrumentos bifaciales hallados en el sitio.

\section{Instrumentos}

Los instrumentos $(\mathrm{n}=18)$ muestran una variedad tecnológica y tipológica que demandó distintos grados de inversión de trabajo. Se pudo reconocer modificaciones en su forma y uso a lo largo de sus historias de vida (Tabla 4). Por ejemplo, los instrumentos no formatizados están representados por cuchillos de filo natural (Figura 2a-c), y se diferencian de un cuchillo de filo retocado marginalmente (Figura 2d). Destaca además, la formatización unifacial e irregular sobre una arista de un percutor elaborado en un fragmento de núcleo (Figura 3a). Otro ejemplo es un fragmento de denticulado en bisel oblicuo/abrupto de sección asimétrica, con microastillamiento unifacial hecho en lasca de jaspe (Figura 3b).

Variabilidad morfológica se observó en varios instrumentos bifaciales. Por ejemplo, una preforma bifacial sugiere un diseño inicial de forma triangular y de base convexa (Figura 4). Puntas de proyectil con estas características se registran en Hakenasa y otros sitios del período Arcaico Temprano de la región (Osorio et al. 2011), por lo que es posible que el tallador tuvo como objetivo producir este modelo de punta triangular.

Tabla 4. Descripción tipológica de los instrumentos analizados de Ipilla 2. Cada categoría de instrumento cuenta con un solo ejemplar, a excepción de los casos donde se indican guarismos mayores.

Typological descriptions of the analyzed tools from Ipilla 2. Each category of instrument has a single specimen, excepting the cases where higher figures are given.

\section{Categorías tipológicas}

Cuchillo de filo natural con rastros complementarios $(\mathrm{N}=3)$.

Cuchillo de filo retocado.

Percutor de arista formatizada sobre núcleo.

Fragmento de denticulado en bisel oblicuo/abrupto de sección asimétrica.

Bifaz.

Preforma de punta de proyectil triangular apedunculada de base convexa.

Punta de proyectil con pedúnculo esbozado, limbo triangular de lados rectos módulo no diferenciado por fractura, pedúnculo de lados rectos convergentes y base convexa inflexionada.

Punta de proyectil con pedúnculo esbozado, limbo triangular corto de lados rectos, pedúnculo de lados rectos convergentes y base no diferenciada por fractura.

Punta de proyectil con pedúnculo destacado y hombros, con limbo triangular corto de bordes denticulados y base convexa. Instrumento compuesto: raspador filo corto + escoplo + cuchillo de filo retocado + raedera + punta burilante.

Cortante sobre punta de proyectil con pedúnculo destacado con hombro, limbo triangular corto con ápice aguzado, con pedúnculo de bordes convergentes convexos y base convexa atenuada.

Fragmentos no diferenciados de artefactos formatizados $(\mathrm{N}=5)$. 
a

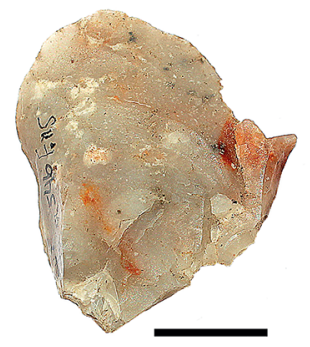

b

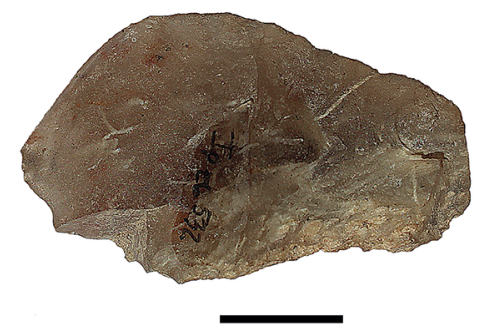

C

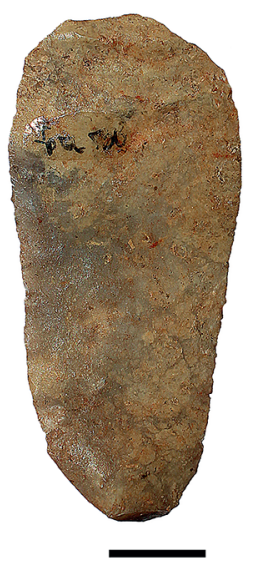

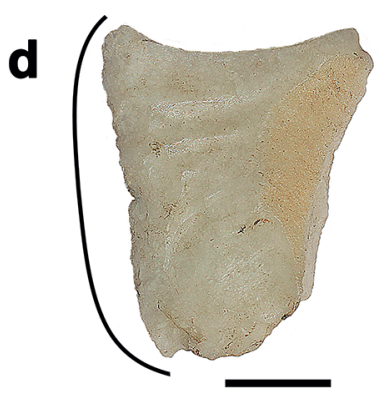

Figura 2. (a, b, y c) Cuchillos de filo natural con rastros complementarios y (d) cuchillo de filo retocado. $(a, b$, and c) Natural edge knives with complementary traces, and (d) retouched edge knife.

a

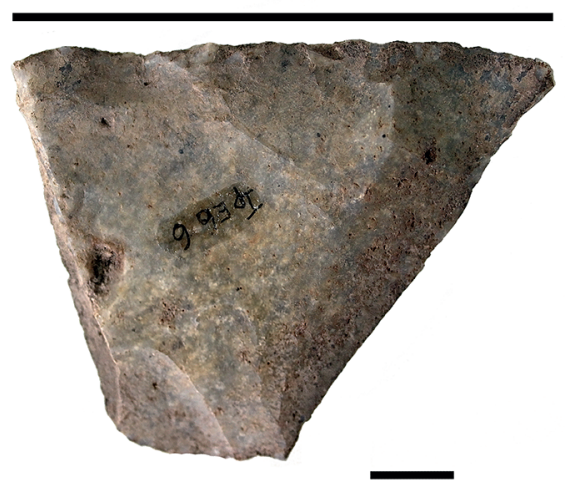

b

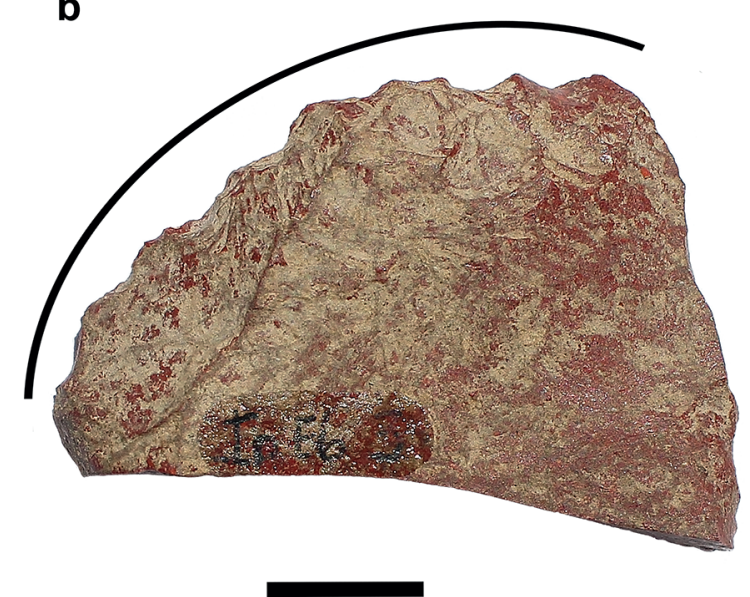

Figura 3. (a) Percutor de arista formatizada sobre núcleo y (b) fragmento de denticulado en bisel oblicuo/abrupto de sección asimétrica. (a) Formalized ridge hammer upon a core, and (b) fragment of denticulated tool with oblique/steep bevel of asymmetrical section.

En otros instrumentos se evidencian los cambios morfológicos a causa de procesos de uso, mantenimiento, re-trabajado y reciclaje. De los tres casos de mantenimiento, los primeros dos corresponden a puntas de proyectil con pedúnculo esbozado de lados rectos convergentes y limbo triangular de lados rectos que muestran mantenimiento diferencial (Figura 5). De base no diferenciada por fractura y limbo triangular corto, la primera punta de proyectil presenta mantenimiento en forma de retoque escamoso escalonado en ambos bordes y caras del limbo, lo que habría provocado pérdida de simetría (Figura 5a). Además, presenta huellas de pulido en los bordes, debido a su uso como proyectil (Lewenstein 1990; Semenov 1981). La segunda punta de proyectil tiene base convexa 


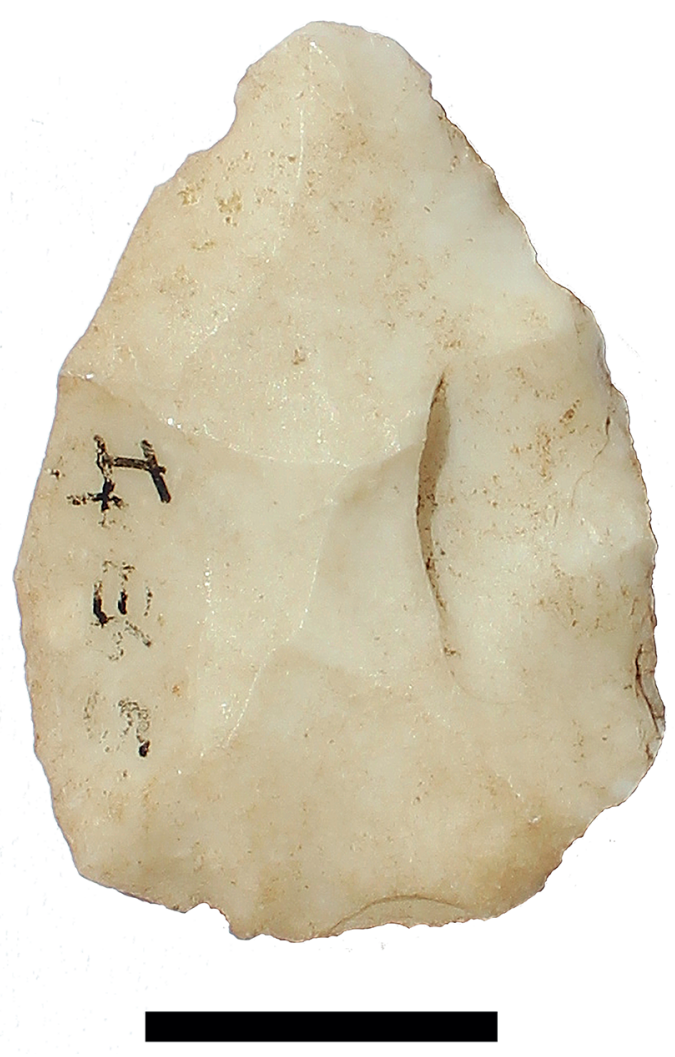

Figura 4. Preforma de punta de proyectil triangular apedunculada de base convexa.

Triangular, non-stemmed projectile point blank with convex base. inflexionada y limbo triangular de módulo no diferenciado, muestra mantenimiento del limbo a través de retoques escamosos marginales y parcialmente extendidos (Figura 5b). Una de sus caras tenía restos de sedimento adheridos lo que limitó evaluar su proceso de formatización. En la base y en parte del pedúnculo, sin embargo, se observó un leve microastillamiento de forma escalonada que pudo ser consecuencia de la tensión del enmangue.

Se observa mantenimiento en una punta de proyectil elaborada sobre una lasca, con pedúnculo destacado y hombros y limbo corto de bordes denticulados. El intenso mantenimiento del limbo provocó la pérdida de su simetría y la disminución de su tamaño, en relación al pedúnculo. A esto se suma un patrón de lascado escamoso escalonado (Figura 6). A pesar de estas modificaciones, se mantuvo el denticulado original de los bordes del limbo. En contraste, en los bordes medial y distal del pedúnculo se observa microastillamiento (parecido a una machacadura), generando un estrechamiento y pérdida de su diseño, posiblemente causado por efecto del enmangue.

Ahora bien, estos tres ejemplares muestran que el diseño original se vio afectado por mantenimiento (pérdida de tamaño, asimetría, cambio en la longitud del limbo, etc.). La morfología de estas puntas de proyectil guarda relación con el tipo conocido como
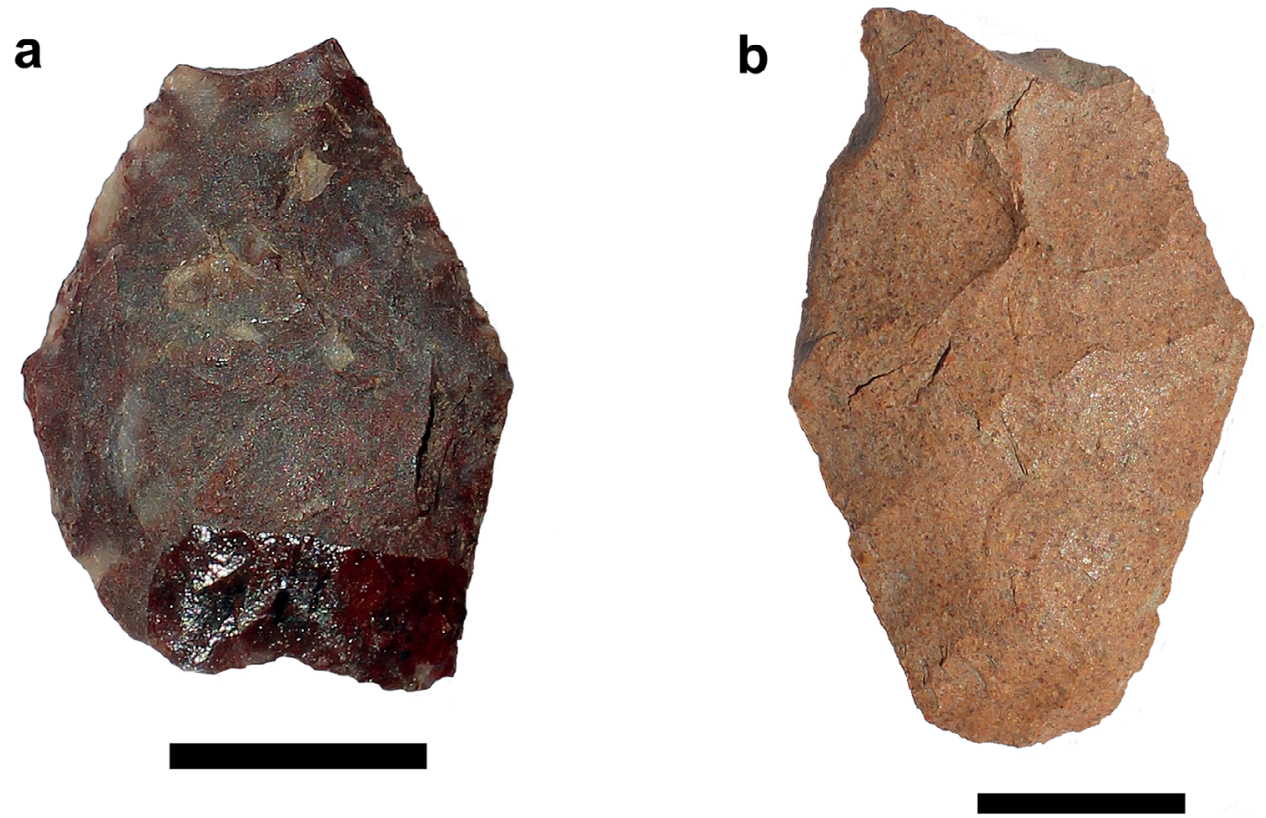

Figura 5. Puntas de proyectil con pedúnculo esbozado: (a) fracturada en ápice y base y (b) fracturada en ápice. Projectile points with outlined stem: (a) tip and base fractured, and (b) tip fractured. 


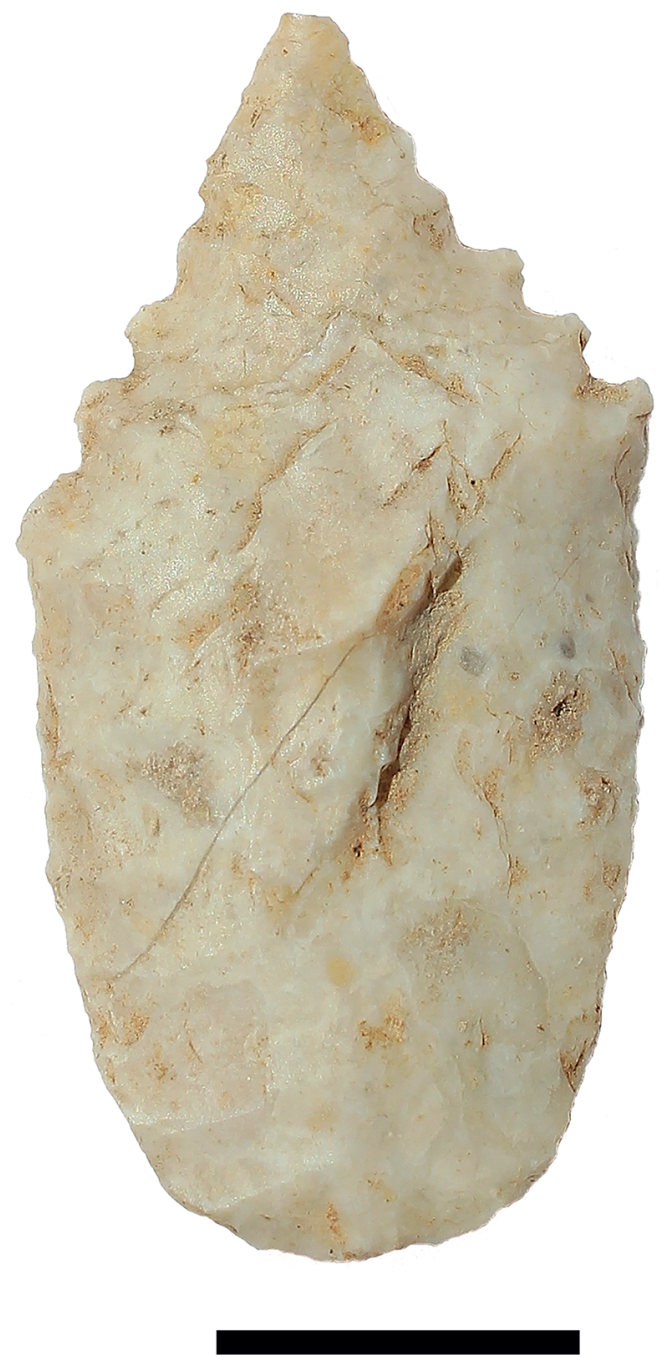

Figura 6. Punta de proyectil con pedúnculo destacado. Projectile point with prominent stem.

Patapatane y que ha sido utilizado como indicador cultural diagnóstico del período Arcaico Temprano de las tierras altas de los Andes Sur Centrales (Klink y Aldenderfer 2005; Osorio et al. 2011; Santoro 1989). El proceso de mantenimiento provocó una serie de transformaciones en el diseño original de las puntas de proyectil de tipo Patapatane, como la reducción de su tamaño, transformación de las aletas en hombros y produciendo un pedúnculo más grande que el limbo.

Se observa mantenimiento y multifuncionalidad en un instrumento de cuatro filos complementarios: raspador, escoplo, cuchillo de filo retocado, raedera y punta burilante (Figura 7). La secuencia de formatización indica que primero fue elaborado el raspador ligeramente convexo (Figura 7a), junto con el escoplo de la parte superior del instrumento (Figura 7b). Ambos filos fueron embotados para formatizar el filo de un cuchillo retocado (Figura 7c) en su borde derecho. Algunos retoques marginales del cuchillo se superponen y se diferencian de los retoques de formatización (paralelos y extendidos) del raspador y del escoplo, lo que significa que este filo fue posterior. El cuarto filo corresponde a una raedera ubicado en el borde izquierdo (Figura 7d) y no presenta superposición de lascado con los otros filos. Al final de la secuencia de formatización, la pieza fue retocada con dos lascados en la parte inferior izquierda, para formar una punta burilante (Figura 7e). Adicionalmente, la cara dorsal del instrumento presenta un dorso pasivo no formatizado que facilitó su prensión digital (Figura 7f).

El instrumento más complejo del conjunto corresponde a un cortante de filo retocado muestra

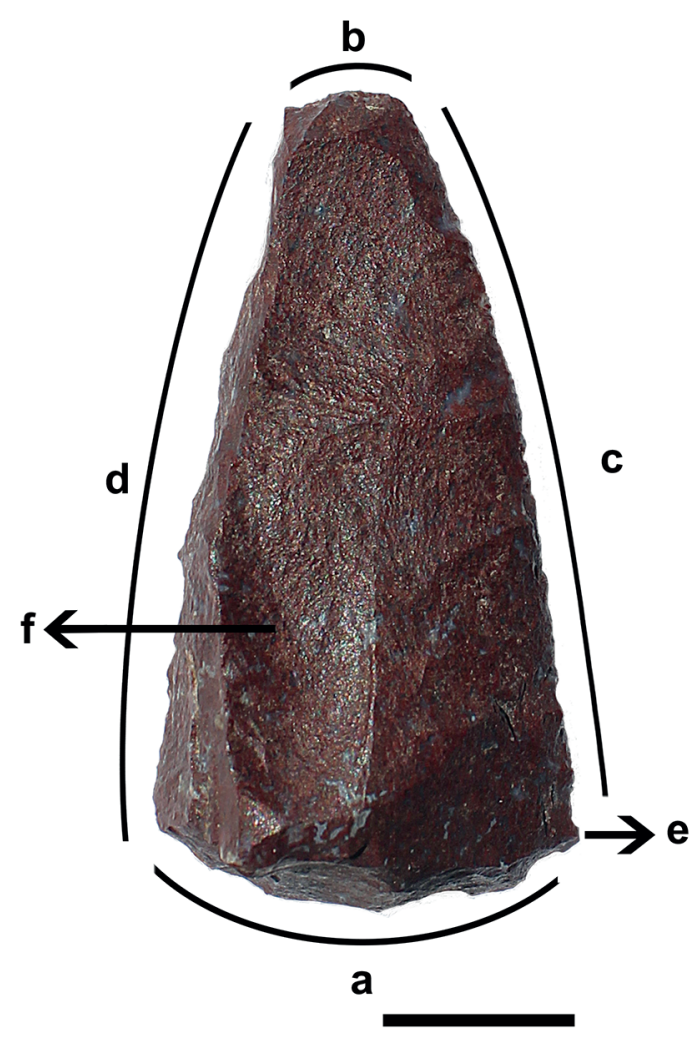

Figura 7. Instrumento compuesto: (a) raspador, (b) escoplo, (c) cuchillo de filo retocado, (d) raedera, (e) punta burilante y, (f) dorso pasivo no formatizado.

Compound artifact: (a) scraper, (b) chisel, (c) retouched edge knife, (d) side-scraper, (e) burin point and, $(f)$ natural back. 


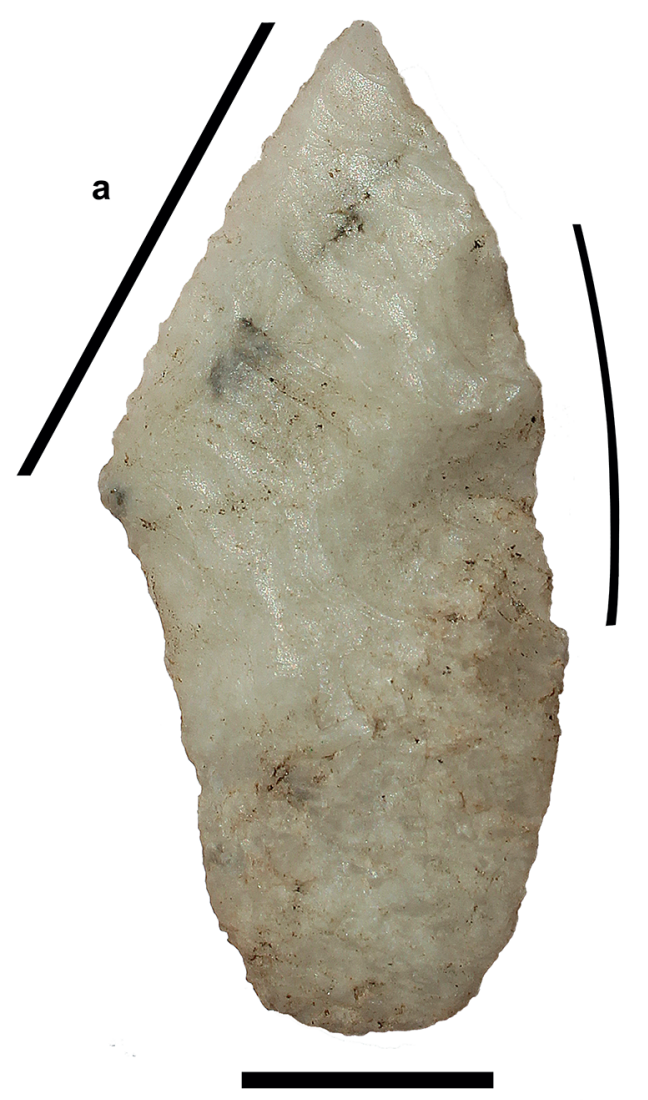

Figura 8. (a) Cortante de filo retocado sobre punta de proyectil reciclada y (b) filo pasivo asociado.

(a) Retouched edge cutter upon recycled projectile point, and (b) related passive edge.

procesos de transformación y reciclaje. Este instrumento fue elaborado sobre una punta de proyectil con pedúnculo destacado de bordes convergentes convexos y un hombro, limbo triangular corto, ápice aguzado y base convexa atenuada (Figura 8). El largo filo del borde activo con huellas de microastillamiento unifacial derivó de acciones de corte (Lewenstein 1990) lo que permite clasificarlo como cortante (Figura 8a). Este reciclaje del filo izquierdo del limbo partió por la transformación del borde derecho a través de dos golpes que eliminaron una aleta y regularizaron su forma alterada por una fractura. De esta manera se conformó un dorso pasivo de $75^{\circ}$ para manipular el cortante (Figura 8b). La transformación morfológica y reciclado de los filos de la punta de proyectil extendió su vida útil pero cambió sus aplicaciones de uso. Adicionalmente, su transformación en un cortante implicó su liberación del astil y el uso manual de la pieza.

\section{Discusión y Conclusiones}

Debido a que los instrumentos líticos son transmisores de información y contienen valores funcionales, prácticos, cognitivos, ideológicos, simbólicos e identitarios, los estudios de tecnología lítica pueden contribuir a mejorar el conocimiento acerca de los cazadores recolectores andinos tempranos (Lemonnier 1992; Sackett 1977, 1983, 1985; Soressi y Geneste 2005; Wiessner 1983; Wobst 1999). La caracterización de tipologías, patrones de transformación, mantenimiento y formas de hacer de los artefactos líticos, permite establecer relaciones tecnológicas entre sitios arqueológicos a través de la inferencia de circuitos de movilidad e interacción regional.

La ubicación de Ipilla 2 en el piso ecológico de puna y sus componentes líticos sugieren que este campamento formaba parte de circuitos de movilidad de cazadores recolectores que arribaron al lugar con sus implementos de trabajo para realizar una serie de actividades. El lugar contaba con vertiente de agua y algunas materias primas de buena calidad como el sílice. Los circuitos de movilidad podrían haber conectado locaciones ubicadas en la zona de puna como Pampa El Muerto-15 (Osorio et al. 2015) y Patapatane (Osorio 2013), dos abrigos con los que podría compartir la misma tecnología lítica. Estos fueron ocupados durante el Arcaico Temprano, cuando las condiciones ambientales eran mejores que las actuales para caza y recolección (Moreno et al. 2009; Mujica et al. 2015; Rech 2001).

En relación a las actividades desarrolladas en Ipilla 2, los instrumentos líticos muestran principalmente acciones de corte para el procesamiento de alimentos. También se infieren actividades especializadas como cacería y el trabajo en cuero y huesos. El tipo de desechos (i.e., desechos de retoque y bifaciales, lascas pequeñas), indica que los instrumentos utilizados fueron reactivados in situ, pero habrían sido elaborados en lugares distintos a la unidad de excavación de Ipilla 2. Procesos más completos de manufactura de artefactos y otro tipo de actividades pudieron ocurrir en sectores no excavados del sitio.

Las diversas formas y procesos técnicos aplicados a los instrumentos muestran distintos grados de inversión de trabajo, mantenimiento y reciclaje, lo que se interpreta como una señal de que los talladores aprovecharon al máximo las formas base, porque sabían distinguir y manejar las potencialidades de 
cada una de las materias primas que utilizaron para conseguir las formas y funciones requeridas. Destaca, por ejemplo, el instrumento compuesto elaborado sobre lasca que estuvo sujeto a una variedad de procesos de talla y uso (Figura 7). En el caso de las puntas de proyectil tipo Patapatane se reveló que su variabilidad morfológica fue consecuencia de una serie de acciones de reciclado para mantener o cambiar su función y alteraron su forma original. El mantenimiento y re-trabajado de éstas y otras puntas de proyectil derivó en las siguientes situaciones: (a) la forma y función del diseño original se mantuvo (Figura 6); (b) el diseño original se transformó, pero su función se mantuvo (Figura 5) y (c) la forma y función del diseño original se modificó (Figura 8). Estos mismos procesos tecnológicos podrían haber afectado a otros tipos de instrumentos como raederas, raspadores y perforadores.

En atención a lo anterior se concluye que las transformaciones morfológicas aplicadas a los artefactos líticos son una señal de la competencia y habilidad de los artesanos de Ipilla para enfrentar problemas como la fractura de una sección del artefacto, el embotamiento de uno de sus filos o la necesidad de darle una nueva función a la pieza en virtud de la circunstancias. Con ello, durante los procesos de elaboración, uso y mantenimiento lograron adecuar los filos y formas de los instrumentos ya fuera para mantener o darle nuevas funciones.

Los procesos de extensión de la vida útil de los instrumentos revelan que sus formas y funciones iniciales pueden cambiar a lo largo de su historia, lo cual confirma nuestra hipótesis de trabajo, que señalaba que las formas de los instrumentos no fueron fijas o estáticas; lo que debería considerarse a la hora de definir una tipología morfológica a un conjunto lítico. Comprender la historia de vida de los artefactos líticos abre camino para reconocer aspectos particulares de los procesos tecnológicos, de las formas de hacer y de utilizar la tecnología. Además de definir las funciones básicas de los instrumentos, el análisis tecno-tipológico permitió explorar la dimensión gestual del uso de los artefactos. Por ejemplo, si éstos fueron tomados por el individuo en forma digital o digito-palmar, o si fueron enmangados y luego liberados del astil. De esta forma, se profundiza el conocimiento acerca de las actividades realizadas en los campamentos de las sociedades de cazadores recolectores que habitaron los ambientes andinos del extremo norte de Chile, durante la transición Pleistoceno Holoceno (ca 10.000-9.000 años a.p.).

Agradecimientos: Estudio financiado por los proyectos Fondecyt N ${ }^{\circ} 1120454$ y 1070140 , donde el primero incluyó la memoria de título de Katherine Herrera que sirvió de base para este manuscrito. Reconocemos el aporte del Centro de Investigaciones del Hombre en el Desierto (CIHDE), CONICYTREGIONAL R07K1001, Proyecto Fondecyt No 3140008 y de la Universidad de Tarapacá a través del Laboratorio de Arqueología y Paleoambiente del Instituto de Alta Investigación. Agradecemos a Marcela Sepúlveda por organizar un taller sobre tecnología lítica en el contexto del proyecto MEC 80120041, a César Méndez, Kurt Rademaker y Vivien G. Standen por su labor editorial y a la/os dos evaluadora/res anónima/os cuyos comentarios ayudaron a mejorar sustancialmente este manuscrito.

\section{Referencias Citadas}

Andrefsky, W. 2005. Lithics: Macroscopic Approaches to Analysis. Segunda edición. Cambridge University Press, Cambridge.

Andrefsky, W. (ed.) 2008. Lithic Technology: Measures of Production, Use, and Curation. Cambridge University Press, Cambridge.

Aschero, C.A. 1975. Ensayo para una clasificación morfológica de artefactos líticos aplicada a estudios tipológicos comparativos. Informe Técnico en posesión de CONICET, Buenos Aires.

Aschero, C.A. 1983. Ensayo para una clasificación morfológica de artefactos líticos aplicada a estudios tipológicos comparativos: Apéndice A-C. Cátedra de Ergología y Tecnología. Facultad de Filosofía y Letras, Universidad de Buenos Aires, Buenos Aires.

Aschero, C.A. y S. Hocsman 2004. Revisando cuestiones tipológicas en torno a la clasificación de artefactos bifaciales.
En Temas de Arqueología: Análisis Lítico, editado por A. Acosta, D. Leponte y M. Ramos, pp. 7-25. Universidad Nacional de Luján, Luján.

Aschero, C.A., S. Hocsman y N. Ratto 2011. Las puntas de proyectil en "mandorla" de Inca Cueva 7: Caracterización tipológica e historia de vida (Puna de Jujuy, Argentina). Estudios Atacameños 41:5-28.

Bate, L.F. 1971. Material lítico: metodología de clasificación. Noticiario Mensual Museo Historia Natural 181:1-23.

Bate, L.F. 1974. Apuntes sobre la Arqueología de los Primeros Poblamientos del Extremo sur Americano. Cuadernos de Trabajo 3, Instituto Nacional de Antropología e Historia, Departamento de Prehistoria, México D.F.

Bird, J. 1965. The concept of a "pre-projectile point" cultural stage in Chile and Peru. American Antiquity 31:262-270. 
Borrazzo, K.B. 2012. Raw material availability, flaking quality, and hunter-gatherer technological decision making in northern Tierra del Fuego Island (southern South America). Journal of Archaeological Science 39:2643-2654.

Brézillon, M. 1983. La Dénomination des Objets de Pierre Taillée. IV Supplément à Gallia Préhistoire, Centre National de la Recherche Scientifique, Paris.

Capriles, J.M., S. Calla Maldonado y J. Albarracin-Jordan 2011. Tecnología lítica y estrategias de subsistencia durante los períodos Arcaico y Formativo en el altiplano central, Bolivia. Chungara Revista de Antropología Chilena 43:455-468.

Cigliano, E. 1962. El Ampajanguense. Universidad Nacional del Litoral, Rosario.

Cruxent, J.M. 1962. Artifacts of paleo-indian type, Maracaibo, Zulia, Venezuela. American Antiquity 27:576-579.

Cruxent, J.M. e I. Rouse 1956. A lithic industry of Paleo-Indian type in Venezuela. American Antiquity 22:172-179.

De Souza, P. 2004. Tecnologías de proyectil durante los períodos Arcaico y Formativo en el Loa Superior (norte de Chile); a partir del análisis de puntas líticas. Chungara Revista de Antropología Chilena 36:61-76.

Dillehay, T.D. 1999. The late Pleistocene cultures of South America. Evolutionary Anthropology 7:206-216.

Dillehay, T.D. 2000. The Settlement of the Americas: A New Prehistory. Basic Books, New York.

Dillehay, T.D., G. Ardila Calderón, G. Politis y M.C. de Moraes Coutinho Beltrão 1992. Earliest hunters and gatherers of South America. Journal of World Prehistory 6:145-204.

Franco, N. 2004. La organización tecnológica y el uso de escalas espaciales amplias. El caso del sur y oeste de Lago Argentino. En Temas de Arqueología: Análisis Lítico, editado por A. Acosta, D. Loponte y M. Ramos, pp. 101-144. Universidad Nacional de Luján, Luján.

González, A.R. 1960. La estratigrafía de la gruta de Intihuasi (Prov. de San Luis, R.A.) y sus relaciones con otros sitios precerámicos de Sudamérica. Revista del Instituto de Antropología 1:5-296.

Hocsman, S. 2006a. Producción Lítica, Variabilidad y Cambio en Antofagasta de la Sierra -ca. 5500-1500 AP-. Tesis Doctoral inédita, Facultad de Ciencias Naturales y Museo, Universidad Nacional de La Plata, La Plata.

Hocsman, S. 2006b. Tecnología lítica en la transición de cazadores recolectores a sociedades agropastoriles en la porción meridional de los Andes Centro Sur. Estudios Atacameños 32:59-73.

Hocsman, S. 2009. Una propuesta de aproximación teóricometodológica a conjuntos de artefactos líticos tallados. En Perspectivas Actuales en Arqueología Argentina, editado por R. Barberena, K. Borrazo y L.A. Borrero, pp. 271-302. Departamento de Investigaciones Prehistóricas y Arqueológicas, IMHICIHU, CONICET, Buenos Aires.

Hocsman, S. 2010. Cambios en las puntas de proyectil durante la transición de cazadores-recolectores a sociedades agro-pastoriles en Antofagasta de la sierra (Puna Argentina). Revista del Instituto de Arqueología Facultad de Filosofía y Letras-UBA 16:59-86.

Hoguin, R. y H.D. Yacobaccio 2012. Análisis lítico de ocupaciones del Holoceno Medio de Hornillos 2 (Jujuy, Argentina): discutiendo la tecnología y distribución de las Puntas de Proyectil "San Martín”. Chungara Revista de Antropología Chilena 44:85-99.

Inizan, M.L., M. Reduron-Ballinger, H. Roche y J. Tixier 1999. Technology and Terminology of Knapped Stone. Préhistoire de la Pierre Taillée Tome 5. Cercle de Recherches et d’Etudes Préhistoriques, Nanterre.

Klink, C. y M.S. Aldenderfer 2005. A proyectile point chronology for the South Central Andean highlands. En Advances in Titicaca Basin Archaeology, editado por C. Stanish, A.B. Cohen y M.S. Aldenderfer, pp. 25-54. Costen Institute of Archaeology, University of California, Los Angeles.

Lanning, E.P. 1973. Burins in the Pleistocene of the Andes. Estudios Atacameños 1:21-37.

Lanning, E.P. y E.A. Hammel 1961. Early lithics industries of western South America. American Antiquity 27:139-154.

Le Paige, G. 1970. Industrias Líticas de San Pedro de Atacama. Editorial Orbe, Santiago.

Lemonnier, P. 1992. Elements for an Anthropology of Technology. Anthropological Papers 88. Museum of Anthropology, University of Michigan, Ann Arbor.

Lewenstein, S. 1990. La función de los artefactos líticos por medio del análisis de huellas de uso. En Nuevos Enfoques en el Estudio de la Lítica, editado, pp. 406-428. Instituto de Investigaciones Antropológicas, Universidad Autónoma de México, México, D.F.

Malinsky-Buller, A., L. Grosman y O. Marder 2011. A case of techno-typological lithic variability \& continuity in the late Lower Palaeolithic. Before Farming 1:1-32.

Mirambell, L. 1994. Fundamentos sobre el estudio de huellas de uso en materiales líticos arqueológicos. Anales de Antropología 31:105-131.

Montane, J. 1972. Las evidencias del poblamiento temprano de Chile. Pumapunku 5:40-53.

Moreno, A., C.M. Santoro y C. Latorre 2009. Climate change and human occupation in the northernmost Chilean Altiplano over the last ca. 11500 cal. a BP. Journal of Quaternary Science 24:373-382.

Mujica, M.I., C. Latorre, A. Maldonado, L. González-Silvestre, R. Pinto, R. De Pol-Holz y C.M. Santoro 2015. Late Quaternary climate change, relict populations and present-day refugia in the northern Atacama Desert: a case study from Quebrada La Higuera (18 S). Journal of Biogeography 42:76-88.

Nelson, M. 1991. The study of technological organization. Archaeological Method and Theory 3:57-100.

Núñez, L. 1983. Paleoindio y Arcaico en Chile: Diversidad, Secuencia y Procesos. Editorial Cuicuilco, México, D.F.

Odell, G.H. (ed.) 1996. Stone Tools Theoretical Insights into Human Prehistory. Plenum Press, New York.

Orquera, L.A. y E.L. Piana 1986. Normas para la descripción de objetos arqueológicos de piedra tallada. Contribución Científica CADIC 1:3-66.

Osorio, D., D. Jackson, P.C. Ugalde, C. Latorre, R. De PolHolz y C.M. Santoro 2011. Hakenasa Cave and its relevance for the peopling of the southern Andean Altiplano. Antiquity 85:1194-1208. 
Osorio, D., M. Sepúlveda, C. Castillo y M. Corvalán 2015. Análisis lítico y funcionalidad de sitios de los aleros de la Precordillera de Arica (Centro-Sur Andino) durante el período Arcaico (ca. 10.000-3700 años a.p.). Intersecciones en Antropología, en prensa.

Ravines, R. 1972. Secuencia y cambios en los artefactos líticos del sur del Perú. Revista del Museo Nacional 38:133-184.

Rech, J.A. 2001. Late Quaternary Paleohydrology and Surficial Processes of the Atacama Desert, Chile: Evidence from Wetland Deposits and Stable Isotopes of Soil Salts. Doctoral Dissertation, Department of Geosciences, University of Arizona, Tucson.

Sackett, J.R. 1977. The meaning of style in archaeology: A general model. American Antiquity 42:369-379.

Sackett, J.R. 1983. Approaches to style in lithic archaeology. Journal of Anthropological Archaeology 1:59-112.

Sackett, J.R. 1985. Style and ethnicity in the Kalahari: A reply to Wiessner. American Antiquity 50:154-159.

Santoro, C.M. 1989. Antiguos cazadores de la puna (9000-6000 a.C.). En Culturas de Chile. Prehistoria, desde sus Orígenes hasta los Albores de la Conquista, editado por J. Hidalgo, V. Schiappacasse, H. Niemeyer, C. Aldunate e I. Solimano, pp. 33-55. Editorial Andrés Bello, Santiago.

Santoro, C.M., C. Latorre, C. Salas, D. Osorio, P. Ugalde, D. Jackson y E.M. Gayó 2011. Ocupación humana pleistocénica en el desierto de Atacama. Primeros resultados de la aplicación de un modelo predictivo interdisciplinario. Chungara Revista de Antropología Chilena 43:353-366.

Santoro, C.M. y L. Núñez 1987. Hunters of the Dry Puna and Salt Puna in northern Chile. Andean Past 1:57-110.

Schlanger, N. 2005. La chaine opératoire. En Archaeology Key Concepts, editado por C. Renfrew y P. Bahn, pp. 433-438. Routledge, London.
Semenov, S. 1981. Tecnología Prehistórica. Estudio de las Herramientas y Objetos Antiguos a Través de las Huellas de Uso. Editorial Akal, Madrid.

Soressi, M. y J.M. Geneste 2005. Discussing the history and the efficacy of the chaine operatoire approach to lithic analysis. A personal view. Ponencia presentada en 71th Annual Meeting of the Society for American Archaeology, San José, Puerto Rico.

Swanson, E.H. (ed.) 1975. Lithic Technology: Making and Using Stone Tools. Mounton Publishers, The Hague.

Taddei, A. 1981. Industrias líticas de Uruguay y su relación con Pampa-Patagonia de Argentina. Revista de Arqueología Americana 3:24-31.

Taddei, A. 1987. Algunos aspectos de la arqueología prehistórica del Uruguay. Estudios Atácamenos 8:62-93.

Ugalde, P.C., C. Salas, C. Latorre, D. Osorio, D. Jackson y C.M. Santoro 2012. Poblamiento temprano del norte de chile (18-25 s): estudio interdisciplinario arqueológico y paleoambiental. Actas XVIII Congreso Nacional de Arqueología Chilena, pp. 197-206. Sociedad Chilena de Arqueología, Santiago.

Wiessner, P. 1983. Style and social information in Kalahari San projectile points. American Antiquity 48:253-276.

Willey, G.R. 1966. An Introduction to American Archaeology, South America. Prentice-Hall, Englewood Cliffs.

Willey, G.R. y P. Phillips 1958. Method and Theory in American Archaeology. University of Chicago Press, Chicago.

Wobst, H.M. 1999. Style in archaeology or archaeologists in style. En Material Meanings: Critical Approaches to the Interpretation of Material Culture, editado por E.S. Chilton, pp. 118-132. University of Utah Press, Salt Lake. 